\title{
OR-54
}

\section{Dentatin Isolated from Clausena Excavata Induces Apoptosis in MCF-7, PC-3 and LNCaP Cells: an Evaluation by the Bioactivity Assay Guided Fractionation}

\author{
Ismail Adam Arbab ${ }^{1, *}$, Ahmad Bustamam Abdul ${ }^{1}$, Mohd Aspollah Sukari $^{2}$, Rasedee Abdullah ${ }^{3}$, \\ Bdria Abdalla Hassan ${ }^{4}$, Suvitha Syam ${ }^{1}$, Mohamed Yousif Ibrahim ${ }^{5}$, Manal Mohamed Elhassan \\ Taha $^{6}$, Siddig Ibrahim Abdelwahab ${ }^{6}$ and Syam Mohan ${ }^{5}$
}

${ }^{1}$ UPM-MAKNA Cancer Research Laboratory, Institute of Bioscience, University Putra Malaysia, Serdang, Selangor,
Malaysia; ${ }^{2}$ Department of Chemistry, Faculty of Science, University Putra Malaysia, Serdang, Selangor, Malaysia;
${ }^{3}$ Department of Veterinary Pathology and Microbiology, Faculty of Veterinary, University Putra Malaysia, Serdang,
Selangor, Malaysia; ${ }^{4}$ Department of Microbiology, Faculty of Veterinary Science, University of Nyala, Nyala, Sudan;
${ }^{5}$ Department of Pharmacy, Faculty of Medicine, University of Malaya, 50603 Kuala Lumpur, Malaysia; ${ }^{6}$ Medical
Research Centre, Jazan University, P.O. Box 114 Jazan, Kingdom of Saudi Arabia;

E-mails: arbabismailadam@yahoo.com; ismailupm2011@gmail.com

Clausena excavata Burm. F., has been used as folk medicines in the eastern of Thailand for the treatment of cancer. Dentatin (DTN) was isolated from this plant via bio assay guided approach and the apoptosis mechanism was investigated. DTN induced cytotoxicity was observed using MTT assay. Acridine orange/Propidium iodide staining was used to detect the early apoptosis cells. High content screening (HCS) was used to observe the nuclear condensation, cell permeability, mitochondrial membrane potential (MMP) and cytochrome c release. Significant increase in chromatin condensation in the cell nucleus was observed in the fluorescent analysis. The apoptosis was confirmed by reduced colony of cells in clonogenic assay and increased cellular DNA breaks on treated cells observed as ladder. Treatment of MCF-7 cells with DTN encouraged apoptosis with cell death-transducing signals that reduced the MMP by down-regulation of Bcl-2 and up-regulation of Bax, triggering the cytochrome $\mathrm{c}$ release from mitochondria to cytosol. Treatment with dentatin dose-dependently inhibited cell growth of PC-3 and LNCaP prostate cancer cell lines, whereas it showed less cytotoxic effects on normal prostate epithelial cell line (RWPE-1). The inhibitory effect of dentatin on prostate cancer cell growth was due to induction of apoptosis as evidenced by Annexin V staining and cell shrinkage. We found that dentatin-mediated accumulation of reactive oxygen species (ROS) and downregulated expression levels of antiapoptotic molecules (Bcl-2, Bcl-xL, and Survivin), leading to disruption of mitochondrial membrane potential (MMP), cell membrane permeability, and release of cytochrome $\mathrm{c}$ from the mitochondria into the cytosol.

Keywords: Clausena excavata, Dentatin, Apoptosis, MMP, Bax/Bcl2. 